\title{
FUZZY IDEALS AND FUZZY DOT IDEALS ON BH-ALGEBRAS
}

\author{
K. Anitha \\ Research Scholar, \\ PG and Research Department of Mathematics, \\ Saiva Bhanu Kshatriya College, \\ Aruppukottai - 626 101, Tamil Nadu, India \\ Dr. N. Kandaraj \\ Associate Professor, \\ PG and Research Department of Mathematics, \\ Saiva Bhanu Kshatriya College, \\ Aruppukottai - 626 101, Tamil Nadu, India
}

\begin{abstract}
In this paper we introduce the notions of Fuzzy Ideals in BH-algebras and the notion of fuzzy dot Ideals of BH-algebras and investigate some of their results.
\end{abstract}

Keywords: BH-algebras, BH- Ideals, Fuzzy Dot BH-ideal.

Cite this Article: K. Anitha and Dr. N. Kandaraj, Fuzzy Ideals and Fuzzy Dot Ideals on Bh-Algebras, International Journal of Advanced Research in Engineering and Technology, 10(2), 2019, pp 350-361.

http://iaeme.com/Home/issue/IJARET?Volume=10\&Issue $=2$

Subject Classification: AMS (2000), 06F35, 03G25, 06D99, 03B47

\section{INTRODUCTION}

Y. Imai and K. Iseki [1, 2, and 3] introduced two classes of abstract algebras: BCK-algebras and BCI-algebras. It is known that the class of BCK-algebras is a proper subclass of the class of BCI-algebras. K. Iseki and S. Tanaka, [7] are introduced Ideal theory of BCK-algebras P. Bhattacharya, N.P. Mukherjee and L.A. Zadeh [4] are introduced fuzzy relations and fuzzy groups. The notion of BH-algebras is introduced by Y. B Jun, E. H. Roh and H. S. Kim[9] Since then, several authors have studied BH-algebras. In particular, Q. Zhang, E. H. Roh and Y. B. Jun [10] studied the fuzzy theory in BH-algebras. L.A. Zadeh [6] introduced notion of fuzzy sets and A. Rosenfeld [8] introduced the notion of fuzzy group. O.G. Xi [5] introduced the notion of fuzzy BCK-algebras. After that, Y.B. Jun and J. Meng [10] studied Characterization of fuzzy sub algebras by their level sub algebras on BCK-algebras. J. Neggers and H. S. Kim[11] introduced on $d$-algebras, M. Akram [12] introduced on fuzzy $d$-algebras In this paper we classify the notion of Fuzzy Ideals on BH - algebras and the notion of Fuzzy dot 


\section{K. Anitha and Dr. N. Kandaraj}

Ideals on $\mathrm{BH}$ - algebras. And then we investigate several basic properties which are related to fuzzy $\mathrm{BH}$-ideals and fuzzy dot $\mathrm{BH}$ - ideals

\section{PRELIMINARIES}

In this section we cite the fundamental definitions that will be used in the sequel:

Definition $2.1[1,2,3]$

Let $\mathrm{X}$ be a nonempty set with a binary operation $*$ and a constant 0 . Then $(\mathrm{X}, *, 0)$ is called a BCK-algebra if it satisfies the following conditions

1. $((x * y) *(x * z)) *(z * y)=0$

2. $(x *(x * y)) * y=0$

$3 . x * x=0$

4. $x * y=0, y * x=0 \Rightarrow x=y$

5. $0 * x=0$ for all $x, y, z \in X$

Definition $2.2[1,2,3]$

Let $\mathrm{X}$ be a BCK-algebra and $\mathrm{I}$ be a subset of $\mathrm{X}$, then $\mathrm{I}$ is called an ideal of $\mathrm{X}$ if

(I1) $0 \in I$

(I2) $y$ and $x * y \in I \Rightarrow x \in I$ for all $x, y \in I$

Definition $2.3[9,10]$

A nonempty set $\mathrm{X}$ with a constant 0 and a binary operation $*$ is called a $\mathrm{BH}$-algebra, if it satisfies the following axioms

(BH1) $x * x=0$

(BH2) $x * 0=0$

(BH3) $x * y=0$ and $y * x=0 \Rightarrow x=y$ for all $x, y \in X$

Example 2.4

Let $X=\{0,1,2\}$ be a set with the following cayley table

\begin{tabular}{|l|l|l|l|}
\hline$*$ & 0 & 1 & 2 \\
\hline 0 & 0 & 1 & 1 \\
\hline 1 & 1 & 0 & 1 \\
\hline 2 & 2 & 1 & 0 \\
\hline
\end{tabular}

Then $(\mathrm{X}, *, 0)$ is a $\mathrm{BH}$-algebra

Definition 2.5[9, 10]

Let $\mathrm{X}$ be a BH-algebra and I be a subset of $\mathrm{X}$, then I is called an ideal of $\mathrm{X}$ if

(BHI) $0 \in I$

(BHI2) $y$ and $x * y \in I \Rightarrow x \in I$

(BHI3) $x \in I$ and $y \in X \Rightarrow x * y \in I$ for all $x, y \in I$

A mapping $f: X \rightarrow Y$ of BH-algebras is called a homomorphism if $f(x * y)=f(x) *$ $f(y)$ for all $x, y X \in X$. Note that if $f: X \rightarrow Y$ is homomorphism of BH-algebras, Then $f(0)=0^{\prime}$. We now review some fuzzy logic concepts. A fuzzy subset of a set $\mathrm{X}$ is a function $\mu: X \rightarrow[0,1]$. For a fuzzy subset $\mu$ of $X$ and $t \in[0,1]$, define $U(\mu ; t)$ to be the set $U(\mu ; t)=$ $\{\mathrm{x} \in \mathrm{X} \mid \mu(\mathrm{x}) \geq \mathrm{t}\}$. For any fuzzy subsets $\mu$ and $v$ of a set $X$, we define

$$
(\mu \cap v)(x)=\min \{\mu(x), v(x)\} \text { for all } x \in X \text {. }
$$


Let $f: X \rightarrow Y$ be a function from a set $\mathrm{X}$ to a set $\mathrm{Y}$ and let $\mu$ be a fuzzy subset of $\mathrm{X}$. The fuzzy subset v of Y defined by $v(y)=\left\{\begin{array}{lll}\sup _{x \in f^{-1}(y)}(x) & \text { if } & f^{-1}(y) \neq \phi, \quad \forall y \in Y \\ & 0 & \text { otherwise }\end{array}\right.$

is called the image of $\mu$ under $f$, denoted by $f(\mu)$. If $v$ is a fuzzy subset of $Y$, the fuzzy subset $\mu$ of $\mathrm{X}$ given by $\mu(x)=v(f(x))$ for all $\mathrm{x} \in \mathrm{X}$ is called the Preimage of $v$ under $\mathrm{f}$ and is denoted by $f^{-1}(v)$. A fuzzy subset $\mu$ in X has the sup property if for any $T \subseteq X$ there exists $x_{0} \in \mathrm{T}$ such that $\mu\left(x_{0}\right)=\sup _{x \in f^{-1}(y)} \mu(z)$. A fuzzy relation $\mu$ on a set $\mathrm{X}$ is a fuzzy subset of $X \times X$, that is, a map $\mu: X \times X \rightarrow[0,1]$.

Definition 2.6[4, 6, 8]

Let $\mathrm{X}$ be a nonempty set. A fuzzy (sub) set $\mu$ of the set $\mathrm{X}$ is a mapping $\mu: X \rightarrow[0,1$

Definition 2.7[4, 6, 8]

Let $\mu$ be the fuzzy set of a set X. For a fixed $s \in[0,1]$, the set $\mu_{s}=\{x \in X: \mu(x) \geq s\}$ is called an upper level of $\mu$ or level subset of $\mu$

Definition $2.8[5,7]$

A fuzzy set $\mu$ in $\mathrm{X}$ is called fuzzy BCK-ideal of $\mathrm{X}$ if it satisfies the following inequalities

1. $\mu(0) \geq \mu(x)$

2. $\mu(x) \geq \min \{\mu(x * y), \mu(y)\}$

Definition 2.9 [11]

Let $\mathrm{X}$ be a nonempty set with a binary operation $*$ and a constant 0 . Then $(\mathrm{X}, *, 0)$ is called a $\mathrm{d}$ - algebra if it satisfies the following axioms.

$1 . x * x=0$

2. $0 * x=0$

3. $x * y=0, y * x=0 \Rightarrow x=y$ for all $x, y \in X$

Definition 2.10 [12]

A fuzzy set $\mu$ in $\mathrm{X}$ is called fuzzy d-ideal of $\mathrm{X}$ if it satisfies the following inequalities

$\operatorname{Fd} 1 . \mu(0) \geq \mu(x)$

$\operatorname{Fd} 2 \mu(x) \geq \min \{\mu(x * y), \mu(y)\}$

Fd3. $\mu(x * y) \geq \min \{\mu(x), \mu(y)\}$ For all $x, y \in X$

Definition 2.11[12] A fuzzy subset $\mu$ of $X$ is called a fuzzy dot $d$-ideal of $X$ if it satisfies

The following conditions:

1. $\mu(0) \geq \mu(x)$

2. $\mu(x) \geq \mu(x * y) \cdot \mu(y)$

3. $\mu(x * y) \geq \mu(x) \cdot \mu(y)$ for all $x, y \in X$

\section{FUZZY IDEALS ON BH-ALGEBRAS}

\section{Definition 3.1}

A fuzzy set $\mu$ in $\mathrm{X}$ is called fuzzy BH-ideal of $\mathrm{X}$ if it satisfies the following inequalities

1. $\mu(0) \geq \mu(x)$

2. $\mu(x) \geq \min \{\mu(x * y), \mu(y)\}$

3. $\mu(x * y) \geq \min \{\mu(x), \mu(y)\}$ for all $x, y \in X$ 


\section{K. Anitha and Dr. N. Kandaraj}

Example 3.2

Let $X=\{0,1,2,3\}$ be a set with the following cayley table

\begin{tabular}{|c|c|c|c|c|}
\hline$*$ & 0 & 1 & 2 & 3 \\
\hline 0 & 0 & 0 & 0 & 0 \\
\hline 1 & 1 & 0 & 0 & 1 \\
\hline 2 & 2 & 2 & 0 & 0 \\
\hline 3 & 3 & 3 & 3 & 0 \\
\hline
\end{tabular}

Then $(\mathrm{X}, *, 0)$ is not BCK-algebra. Since $\{(1 * 3) *(1 * 2)\} *(2 * 3)=1 \neq 0$

We define fuzzy set $\mu$ in $\mathrm{X}$ by $\mu(0)=0.8$ and $\mu(x)=0.01$ for all $x \neq 0$ in $\mathrm{X}$. then it is easy to show that $\mu$ is a $\mathrm{BH}$-ideal of $\mathrm{X}$.

We can easily observe the following propositions

1. In a BH-algebra every fuzzy BH-ideal is a fuzzy BCK-ideal, and every fuzzy BCKideal is a fuzzy BH -Sub algebra

2. Every fuzzy BH-ideal of a BH- algebra is a fuzzy BH-subalgebra.

Example 3.3

Let $X=\{0,1,2\}$ be a set given by the following cayley table

\begin{tabular}{|c|c|c|c|}
\hline$*$ & 0 & 1 & 2 \\
\hline 0 & 0 & 0 & 0 \\
\hline 1 & 1 & 0 & 1 \\
\hline 2 & 2 & 2 & 0 \\
\hline
\end{tabular}

Then $(\mathrm{X}, *, 0)$ is a fuzzy BCK-algebra. We define fuzzy set $\mu$ in $\mathrm{X}$ by $\mu(0)=0.7, \mu(1)=$ $0.5, \mu(2)=0.2$

Then $\mu$ is a fuzzy BH-ideal of $\mathrm{X}$.

\section{Definition 3.4}

Let $\lambda$ and $\mu$ be the fuzzy sets in a set $X$. The Cartesian product $\lambda \times \mu: X \times X \rightarrow[0,1]$ is defined by $(\lambda \times \mu)(x, y)=\min \{\lambda(x), \mu(y)\} \forall x, y \in X$.

\section{Theorem 3.5}

If $\lambda$ and $\mu$ be the fuzzy BH-ideals of a BH- algebra $\mathrm{X}$, then $\lambda \times \mu$ is a fuzzy BH-ideals of $X \times X$

\section{Proof}

For any $(x, y) \in X \times X$, wehave

$(\lambda \times \mu)(0,0)=\min \{\lambda(0), \mu(0)\} \geq \min \{\lambda(x), \mu(y)\}$

$$
=(\lambda \times \mu)(x, y)
$$

That is $(\lambda \times \mu)(0,0)=(\lambda \times \mu)(x, y)$

Let $\left(x_{1}, x_{2}\right)$ and $\left(y_{1}, y_{2}\right) \in X \times X$

Then, $(\lambda \times \mu)\left(x_{1}, x_{2}\right)=\min \left\{\lambda\left(x_{1}\right), \mu\left(x_{2}\right)\right\}$

$\geq \min \left\{\min \left\{\lambda\left(x_{1} * y_{1}\right), \lambda\left(y_{1}\right)\right\}, \min \left\{\mu\left(x_{2} * y_{2}\right), \mu\left(y_{2}\right)\right\}\right.$

$=\min \left\{\min \lambda\left(x_{1} * y_{1}\right), \mu\left(x_{2} * y_{2}\right), \min \left\{\lambda\left(y_{1}\right), \mu\left(y_{2}\right)\right\}\right\}$

$\left.=\min \left\{(\lambda \times \mu)\left(x_{1} * y_{1}, x_{2} * y_{2}\right)\right),(\lambda \times \mu)\left(y_{1}, y_{2}\right)\right\}$

$=\min \left\{\left((\lambda \times \mu)\left(x_{1}, x_{2}\right) *\left(y_{1}, y_{2}\right)\right),(\lambda \times \mu)\left(y_{1}, y_{2}\right)\right\}$

That is $\left.\left((\lambda \times \mu)\left(x_{1}, x_{2}\right)\right)=\min \left\{(\lambda \times \mu)\left(x_{1}, x_{2}\right) *\left(y_{1}, y_{2}\right)\right),(\lambda \times \mu)\left(y_{1}, y_{2}\right)\right\}$

And $(\lambda \times \mu)\left(\left(x_{1}, x_{2}\right) *\left(y_{1}, y_{2}\right)\right)$ 
$=(\lambda \times \mu)\left(x^{1} * y^{1}, x^{2} * y^{2}\right)$

$=\min \left\{\lambda\left(x_{1} * y_{1}\right), \mu\left(x_{2} * y_{2}\right)\right\}$

$\geq \min \left\{\min \left\{\lambda\left(x_{1}\right), \lambda\left(y_{1}\right)\right\}, \min \left\{\mu\left(x_{2}\right), \mu\left(y_{2}\right)\right\}\right\}$

$=\min \left\{\min \left(\lambda\left(x_{1}\right), \mu\left(x_{2}\right), \min \left\{\left(\lambda\left(y_{1}\right), \mu\left(y_{2}\right)\right)\right\}\right.\right.$

$=\min \left\{(\lambda \times \mu)\left(\left(x_{1}, x_{2}\right),(\lambda \times \mu)\left(y_{1}, y_{2}\right)\right)\right.$

That is $(\lambda \times \mu)\left(\left(x_{1}, x_{2}\right) *\left(y_{1}, y_{2}\right)\right)$

$=\min \left\{(\lambda \times \mu)\left(\left(x_{1}, x_{2}\right),(\lambda \times \mu)\left(y_{1}, y_{2}\right)\right\}\right.$

Hence $\lambda \times \mu$ is a fuzzy BH-ideal of $\mathrm{X} \times X$

Theorem 3.6

Let $\lambda$ and $\mu$ be fuzzy sets in a BH-algebra such that $\lambda \times \mu$ is a fuzzy $\mathrm{BH}$-ideal of $X \times X$.Then

i)Either $\lambda(0) \geq \lambda(\mathrm{x})$ or $\mu(0) \geq \mu(x) \forall x \in X$.

ii) If $\lambda(0) \geq \lambda(\mathrm{x}) \forall x \in X$, then either $\mu(0) \geq \lambda(x)$ or $\mu(0) \geq \mu(x)$

iii) If $\mu(0) \geq \mu(x) \forall x \in X$, then either $\lambda(0) \geq \lambda$ (x) or $\lambda(0) \geq \mu(\mathrm{x})$

\section{Proof}

We use reduction to absurdity

i) Assume $\lambda(\mathrm{x})>\lambda(0)$ and $\mu(x) \geq \mu(0)$ for some $x, y \in X$.

Then $(\lambda \times \mu)(x, y)=\min \{\lambda(\mathrm{x}), \mu(y)\}$

$$
\begin{aligned}
& >\min \{\{\lambda(0), \mu(0)\} \\
& =(\lambda \times \mu)(0,0)
\end{aligned}
$$

$(\lambda \times \mu)(x, y)>(\lambda \times \mu)(0,0) \forall x, y \in X$

Which is a contradiction to $(\lambda \times \mu)$ is a fuzzy BH-ideal of $\mathrm{X} \times X$

Therefore either $\lambda(0) \geq \lambda(\mathrm{x}) \operatorname{or} \mu(0) \geq \mu(x) \forall x \in X$.

ii) Assume $\mu(0)<\lambda(x)$ and $\mu(0)<\mu(y)$ for some $x, y \in X$.

Then $(\lambda \times \mu)(0,0)=\min \{\lambda(0), \mu(0)\}=\mu(0)$

And $(\lambda \times \mu)(x, y)=\min \{\lambda(\mathrm{x}), \mu(y)\}>\mu(0)$

$$
=(\lambda \times \mu)(0,0)
$$

This implies $(\lambda \times \mu)(x, y)>$ and $(\lambda \times \mu)(0,0)$

Which is a contradiction to $\lambda \times \mu$ is a fuzzy BH-ideal of $\mathrm{X} \times X$

Hence if $\lambda(0) \geq \lambda(\mathrm{x}) \forall x \in X$, then

Either $\mu(0) \geq \lambda(x)$ or $\mu(0) \geq \mu(x) \quad \forall x \in X$

iii) Assume $\lambda(0)<\lambda(\mathrm{x})$ or $\lambda(0)<\mu(\mathrm{y}) \forall x, y \in X$

Then $((\lambda \times \mu)(0,0)=\min \{\lambda(0), \mu(0)\}=\lambda(0)$

And $(\lambda \times \mu)(x, y)=\min \{\lambda(\mathrm{x}), \mu(y)\}>\lambda(0)$

$$
=(\lambda \times \mu)(0,0)
$$

This implies $(\lambda \times \mu)(x, y)>(\lambda \times \mu)(0,0)$

Which is a contradiction to $(\lambda \times \mu)$ is a fuzzy BH-ideal of $X \times X$

Hence if $\mu(0) \geq \mu(x) \quad \forall x \in X$ then either $\lambda(0) \geq \lambda(\mathrm{x})$ or $\lambda(0) \geq \mu(\mathrm{x})$

This completes the proof

\section{Theorem 3.7}

If $\lambda \times \mu$ is a fuzzy BH-idela of $X \times X$ then $\lambda$ or $\mu$ is a fuzzy BH-ideal of $\mathrm{X}$. 


\section{Proof}

First we prove that $\mu$ is a fuzzy BH-ideal of $\mathrm{X}$.

Given $\lambda \times \mu$ is a fuzzy BH-ideal of $X \times X$, then by theorem 3.6(i), either $\lambda(0) \geq$ $\lambda(\mathrm{x}) \operatorname{or} \mu(0) \geq \mu(x) \forall x \in X$.

Let $\mu(0) \geq \mu(x)$

By theorem 3.6(iii) then either $\lambda(0) \geq \lambda(\mathrm{x})$ or $\lambda(0) \geq \mu(\mathrm{x})$

$$
\text { Now } \begin{aligned}
\mu(x) & =\min \{\lambda(0), \mu(x)\} \\
& =(\lambda \times \mu)(0, x) \\
& \geq \min \{((\lambda \times \mu)(0, x) *(0, y)),(\lambda \times \mu)(0, y)\} \\
& =\min \{(\lambda \times \mu)(0 * 0), x * y),(\lambda \times \mu)(0, y)\} \\
& =\min \{(\lambda \times \mu)(0, x * y),(\lambda \times \mu)(0, y)\} \\
& =\min \{(\lambda \times \mu)(0 * 0), x * y),(\lambda \times \mu)(0, y)\} \\
& =\min \{\mu(x * y),(\mu)(y)\}
\end{aligned}
$$

That is $\mu(x) \geq \min \{\mu(x * y), \mu(y)\}$

$$
\begin{aligned}
\mu(x * y) & =\min \{\lambda(0), \mu(x * y)\} \\
& =(\lambda \times \mu)(0, x * y) \\
& =(\lambda \times \mu)(0 * 0, x * y) \\
& =(\lambda \times \mu)(0, x) *(0, y) \\
\mu(x * y) & \geq \min \{(\lambda \times \mu)(0, x),(\lambda \times \mu)(0, y)\} \\
& =\min \{\mu(x), \mu(y)\}
\end{aligned}
$$

That is, $\mu(x * y) \geq \min \{\mu(x), \mu(y)\}$

This proves that $\mu$ is a fuzzy BH-ideal of $\mathrm{X}$.

Secondly to prove that $\lambda$ is a Fuzzy BH-ideal of $\mathrm{X}$.

Using theorem 4.6(i) and (ii) we get

This completes the proof.

\section{Theorem 3.8}

If $\mu$ is a fuzzy BH-idela of $X$, then $\mu_{t}$ is a BH-idela of $\mathrm{X}$ for all $t \in[0,1]$

\section{Proof}

Let $\mu$ be a fuzzy BH-ideal of $\mathrm{X}$,

Then By the definition of BH-ideal

$$
\mu(0) \geq \mu(x)
$$

$\mu(x) \geq \min \{\mu(x * y),(\mu(y)\}$

$\mu(x * y) \geq \min \{\mu(x),(\mu(y)\} \quad \forall x, y \in X$

To prove that $\mu_{t}$ is a $\mathrm{BH}$-ideal of $\mathrm{x}$.

By the definition of level subset of $\mu$

$\mu_{t}=\{x / \mu(x) \geq t\}$

Let $x, y \in \mu_{t}$ and $\mu$ is a fuzzy BH-ideal of $\mathrm{X}$.

Since $\mu(0) \geq \mu(x) \geq t$ implies $0 \in \mu_{t}$, for all $t \in[0,1]$

Let $x, y \in \mu_{t}$ and $y \in \mu_{t}$

Therefore $\mu(x * y) \geq t$ and $\mu(y) \geq t$

Now $\mu(x) \geq \min \{\mu(x * y),(\mu(y)\}$ 
$\geq \min \{t, t\} \geq t$

Hence $(\mu(x) \geq t$

That is $x \in \mu_{t}$.

Let $x \in \mu_{t}, y \in X$

Choose $\mathrm{y}$ in $\mathrm{X}$ such that $\mu(y) \geq t$

Since $x \in \mu_{t}$ implies $\mu(x) \geq t$

We know that $\mu(x * y) \geq \min \{\mu(x),(\mu(y)\}$

$$
\begin{aligned}
& \geq \min \{t, t\} \\
& \geq t
\end{aligned}
$$

That is $\quad \mu(x * y) \geq t$ implies $x * y \in \mu_{t}$

Hence $\mu_{t}$ is a BH-ideal of $\mathrm{X}$.

\section{Theorem 3.9} $\mathrm{X}$.

If $\mathrm{X}$ be a BH-algebra, $\forall t \in[0,1]$ and $\mu_{t}$ is a $B H$-ideal of $\mathrm{X}$, then $\mu$ is a fuzzy BH-ideal of

\section{Proof}

Since $\mu_{t}$ is a $B H$-ideal of $\mathrm{X}$

$0 \in \mu_{t}$

ii) $x * y \in \mu_{t}$ And $y \in \mu_{t}$ implies $x \in \mu_{t}$

iii) $x \in \mu_{t}, \mathrm{y} \in X$ implies $x * y \in \mu_{t}$

To prove that $\mu$ is a fuzzy $\mathrm{BH}$-ideal of $\mathrm{X}$.

Let $x, y \in \mu_{t}$ then $\mu(x) \geq t$ and $\mu(y) \geq t$

Let $\mu(x)=t_{1}$ and $\mu(y)=t_{2}$

Without loss of generality let $t_{1} \leq t_{2}$

Then $x \in \mu_{t_{1}}$

Now $x \in \mu_{t_{1}}$ and $\mathrm{y} \in X$ implies $x * y \in \mu_{t_{1}}$

That is $\mu(x * y) \geq t_{1}$

$$
\begin{aligned}
& =\min \left\{t_{1}, t_{2}\right\} \\
& =\min \{\mu(x), \mu(y)\} \\
\mu(x * y) & \geq \min \{\mu(x), \mu(y)\}
\end{aligned}
$$

ii) Let $\mu(0)=\mu(x * x) \geq \min \{\mu(x), \mu(y)\} \geq \mu(x)$ (by proof (i))

That is $\mu(0) \geq \mu(x)$ for all $\mathrm{x} \in X$

iii) Let $\mu(x)=\mu(x * y) *(0 * y))$

$$
\begin{aligned}
& \geq \min \{\mu(x * y), \mu(0 * y))(\mathrm{By}(\mathrm{i})) \\
& \geq \min \{\mu(x * y), \min \{\mu(0), \mu(y)\} \\
& \geq \min \{\mu(x * y),\{\mu(y))(\mathrm{By}(\mathrm{ii})) \\
\mu(x) & \geq \min \{\mu(x * y),\{\mu(y))
\end{aligned}
$$

Hence $\mu$ is a fuzzy $\mathrm{BH}$-ideal of $\mathrm{X}$.

Definition 3.10

A fuzzy set $\mu$ in $\mathrm{X}$ is said to be fuzzy BH- $\chi$ ideal if $\mu(x * u * v * y) \geq \min \{\mu(x), \mu(y)\}$ 


\section{Theorem 3.11}

Every Fuzzy BH -ideal is a fuzzy BH- $\boldsymbol{\chi}$ - ideal

\section{Proof}

It is trivial

\section{Remark}

Converse of the above theorem is not true. That is every fuzzy BH- $\chi$-ideal is not true. That is every fuzzy $\mathrm{BH}$-ideal. Let us prove this by an example

Let $X=\{0,1,2\}$ be a set given by the following cayley table

\begin{tabular}{|c|c|c|c|}
\hline$*$ & 0 & 1 & 2 \\
\hline 0 & 0 & 1 & 2 \\
\hline 1 & 1 & 0 & 1 \\
\hline 2 & 2 & 2 & 0 \\
\hline
\end{tabular}

Then $(\mathrm{X}, *, 0)$ is a $\mathrm{BH}$-algebra. We define fuzzy set: $\mathrm{X} \rightarrow[0,1]$ by $\mu(0)=0.8, \mu(x)=$ $0.2 \forall x \neq 0$ clearly $\mu$ is a fuzzy BH-ideal of X But $\mu$ is not a BH- $\chi$-ideal of $\mathrm{X}$.

For Let $x=0 u=1 \quad v=1 y=1$

$$
\begin{aligned}
& \mu(x * u * v * y=\mu(0 * 1 * 1 * 1)=\mu(1)=0.2 \\
& \min \{\mu(x), \mu(y)\}=\min \{\mu(0), \mu(0)\} \\
& =\mu(0)=0.8 \\
& \mu(x * u * v * y) \leq \min \{\mu(x), \mu(y)\}
\end{aligned}
$$

Hence $\mu$ is not a fuzzy BH- $\chi$-ideal of X.

And

$$
\begin{aligned}
\mu(x * y) & =\mu(f(a) * f(b)) \\
& \geq \mu(f(a * b)) \\
& =\mu^{f}(a * b) \\
& \geq \min \left\{\mu^{f}(a), \mu^{f}(b)\right\} \\
& =\min \{\mu(f(a), \mu(f(b))\} \\
& =\min \{\mu(x), \mu(y)\}
\end{aligned}
$$

Hence $\mu(x * y) \geq \min \{\mu(x), \mu(y)\}$

Hence $\mu$ is a fuzzy BH- $\chi$ ideal of Y.

\section{FUZZY DOT BH-IDEALS OF BH-ALGEBRAS}

Definition 4.1. A fuzzy subset $\mu$ of $X$ is called a fuzzy dot $B H$-ideal of $X$ if it satisfies

The following conditions:

(FBH1). $\mu(0) \geq \mu(x)$

(FBH2). $\mu(x) \geq \mu(x * y) \cdot \mu(y)$

(FBH3). $\mu(x * y) \geq \mu(x) . \mu(y)$ for all $x, y \in X$

Example 4.2. Let $X=\{0,1,2,3\}$ be a $B H$-algebra with Cayley table (Table 1) as follows:

(Table 1)

\begin{tabular}{|c|c|c|c|c|}
\hline$*$ & 0 & 1 & 2 & 3 \\
\hline 0 & 0 & 0 & 0 & 0 \\
\hline 1 & 1 & 0 & 0 & 2 \\
\hline 2 & 2 & 2 & 0 & 0 \\
\hline 3 & 3 & 3 & 3 & 0 \\
\hline
\end{tabular}


Define $\mu: X \rightarrow[0,1]$ by $\mu(0)=0.9, \mu(a)=\mu(b)=0.6, \mu(c)=0.3$.It is easy to verify that $\mu$ is a Fuzzy dot $B H$-ideal of $X$.

Proposition 4.3. Every fuzzy $B H$-ideal is a fuzzy dot $B H$-ideal of a $B H$-algebra.

Remark. The converse of Proposition 4.3 is not true as shown in the following Example 4.2. Let $X=\{0,1,2,3\}$ be a $B H$-algebra with Cayley table (Table 1) as follows:

Example 4.4. Let $X=\{0,1,2\}$ be a $B H$-algebra with Cayley table (Table 2) as follows:

(Table 2)

\begin{tabular}{|c|c|c|c|}
\hline$*$ & 0 & 1 & 2 \\
\hline 0 & 0 & 0 & 0 \\
\hline 1 & 1 & 0 & 2 \\
\hline 2 & 2 & 1 & 0 \\
\hline
\end{tabular}

Define $\mu: X \rightarrow[0,1]$ by $\mu(0)=0.8, \mu(1)=0.5, \mu(2)=0.4$. It is easy to verify that $\mu$ is a fuzzy Dot $B H$-ideal of $X$, but not a fuzzy $d$-ideal of $X$ because

$$
\begin{aligned}
\mu(x) & \leq \min \{\mu(x * y), \mu(y)\} \\
\mu(1) & =\min \{\mu(1 * 2), \mu(2)\} \\
& =\mu(2)
\end{aligned}
$$

Proposition 4.5. Every fuzzy dot $B H$-ideal of a $B H$-algebra $X$ is a fuzzy dot subalgebra of $X$.

Remark. The converse of Proposition 4.5 is not true as shown in the following

Example:

Example 4.6. Let $X$ be the BH-algebra in Example 4.4 and define $\mu: X \rightarrow[0,1]$ by $\mu(0)=\mu(1)=0.9, \mu(2)=0.7$. It is easy to verify that $\mu$ is a fuzzy dot sub algebra of $X$, but not a fuzzy dot $B H$-ideal of $X$ because $\mu(2)=0.7 \leq 0.81=\mu(2 * 1) \cdot \mu(1)$.

Proposition 4.7. If $\mu$ and $\nu$ are fuzzy dot $B H$-ideals of a $B H$-algebra $X$, then so is $\mu \cap \nu$. Proof. Let $x, y \in X$. Then

$$
\begin{gathered}
(\mu \cap v)(0)=\min \{\mu(0), v(0)\} \\
\geq \min \{\mu(x), v(x)\} \\
=(\mu \cap v)(x)
\end{gathered}
$$

Also, $(\mu \cap v)(x)=\min \{\mu(x), v(x)\}$

$$
\begin{gathered}
\geq \min \{\mu(x * y) \cdot \mu(y), v(x * y) \cdot v(y)\} \\
\geq(\min \{\mu(x * y), v(x * y)\}) \cdot(\min \{\mu(y), v(y)\}) \\
=((\mu \cap v)(x * y)) \cdot((\mu \cap v)(y)) .
\end{gathered}
$$$$
\text { And, }(\mu \cap v)(x * y)=\min \{\mu(x * y), v(x * y)\}
$$$$
\geq \min \{\mu(x) \cdot \mu(y), v(x) \cdot v(y)\}
$$$$
\geq(\min \{\mu(x), v(x)\}) \cdot(\min \{\mu(y), v(y)\})
$$$$
=((\mu \cap v)(x)) \cdot((\mu \cap v)(y)) \text {. }
$$

Hence $\mu \cap v$ is a fuzzy dot $B H$-ideal of a $d$-algebra $X$.

Theorem 4.8. If each nonempty level subset $U(\mu ; t)$ of $\mu$ is a fuzzy $B H$-ideal of $X$ then $\mu$ is a fuzzy dot $B H$-ideal of $X$, where $t \in[0,1]$. 


\section{Definition 4.9}

Let $\sigma$ be a fuzzy subset of $X$. The strongest fuzzy $\sigma$-relation on BH-algebra $\mathrm{X}$ is the fuzzy subset $\mu_{\sigma}$ of $\mathrm{X} \times \mathrm{X}$ given by $\mu_{\sigma}(\mathrm{x}, \mathrm{y})=\sigma(\mathrm{x}) \cdot \sigma(\mathrm{y})$ for all $\mathrm{x}, \mathrm{y} \in \mathrm{X}$. A fuzzy relation $\mu$ on $\mathrm{BH}-$ algebra $\mathrm{X}$ is called a Fuzzy $\sigma$-product relation if $\mu(\mathrm{x}, \mathrm{y}) \geq \sigma(\mathrm{x}) \cdot \sigma(\mathrm{y})$ for all $\mathrm{x}, \mathrm{y} \in \mathrm{X}$. A fuzzy relation $\mu$ on $\mathrm{BH}$-algebra is called a left fuzzy relation on $\sigma$ if $\mu(\mathrm{x}, \mathrm{y})=\sigma(\mathrm{x})$ for all $\mathrm{x}, \mathrm{y}$ $\in X$.

Note that a left fuzzy relation on $\sigma$ is a fuzzy $\sigma$-product relation.

Remark. The converse of Theorem 4.8 is not true as shown in the following example:

Example 4.10. Let $X$ be the $B H$-algebra in Example 4.4 and define $\mu: \mathrm{X} \rightarrow[0,1]$ by

$\mu(0)=0.6, \mu(1)=0.7, \mu(2)=0.8$. We know that $\mu$ is a fuzzy $\operatorname{dot} B H$-ideal of $X$, but $U(\mu ; 0.8)=\{x \in X \mid \mu(x) \geq 0.8\}=\{2,2\}$ is not $B H$-ideal of $X$ since $0 \notin U(\mu ; 0.8)$.

Theorem 4.11. Let $f: X \rightarrow X^{\prime}$ be an onto homomorphism of $B H$-algebras, $v$ be a fuzzy Dot BH-ideal of $Y$. Then the Preimage $f^{-1}(v)$ of $v$ under $f$ is a fuzzy dot $B H$-ideal of $X$.

Proof. Let $x \in X$,

$$
\begin{aligned}
& f^{-1}(v)(0)=v(f(0))=v\left(0^{\prime}\right) \\
& \geq v(f(x))=f^{-1}(v)(x)
\end{aligned}
$$

For any $x, y \in X$, we have

$f^{-1}(v)(x)=v(\mathrm{f}(\mathrm{x})) \geq v(f(x) * f(y)) \cdot v(f(y))$

$=v\left(f\left(x^{*} y\right)\right) \cdot v(f(y))=f^{-1}(v)(x * y) \cdot f^{-1}(v)(y)$

Also,

$f^{-1}(v)(x * y)=v(f(x * y))=v(f(x) * f(y))$

$\geq v(f(x)) \cdot v(f(y))=f^{-1}(v)(x) \cdot f^{-1}(v)(y)$

Thus $f^{-1}(v)$ is a fuzzy dot $B H$-ideal of $X$.

Theorem: 4.12 An onto homomorphic image of a fuzzy dot $B H$-ideal with the sup Property is a fuzzy dot $\mathrm{BH}$-ideal.

Theorem 4.13. If $\lambda$ and $\mu$ are fuzzy dot $B H$-ideal of a $B H$-algebra $X$, then $\lambda \times \mu$ is a fuzzy Dot $B H$-ideal of $X \times X$.

Proof.

Let $x, y \in X$

$\lambda \times \mu(0,0)=\lambda(0), \mu(0)$

$\geq \lambda(\mathrm{x}) \cdot \mu(\mathrm{y})=(\lambda \times \mu)(\mathrm{x}, \mathrm{y})$

For any $x, x^{\prime}, y, y^{\prime} \in X$ wehave

$(\lambda \times \mu)(\mathrm{x}, \mathrm{y})=\lambda(\mathrm{x}) \cdot \mu(\mathrm{y})$

$\left.\geq \lambda\left(\mathrm{x} * \mathrm{x}^{\prime}\right) \cdot \lambda\left(\mathrm{x}^{\prime}\right)\right) \mu\left(\mathrm{y} * \mathrm{y}^{\prime}\right) \cdot \mu\left(\mathrm{y}^{\prime}\right)$

$\left.=\lambda\left(\mathrm{x} * \mathrm{x}^{\prime}\right) \cdot \mu\left(\mathrm{y} * \mathrm{y}^{\prime}\right)\right) \cdot \lambda\left(\mathrm{x}^{\prime}\right) \cdot \mu\left(\mathrm{y}^{\prime}\right)$

$\left.=(\lambda \times \mu)(\mathrm{x}, \mathrm{y}) *\left(\mathrm{x}^{\prime}, \mathrm{y}^{\prime}\right)\right) \cdot(\lambda \times \mu)\left(\mathrm{x}^{\prime}, \mathrm{y}^{\prime}\right)$

Also $\left.\left.(\lambda \times \mu)(x, y) *\left(x^{\prime}, y^{\prime}\right)\right)=(\lambda x \mu)\left(x * x^{\prime}\right) *\left(y * y^{\prime}\right)\right)$.

$=\lambda\left(\mathrm{x} * \mathrm{x}^{\prime}\right) \cdot \mu\left(\mathrm{y} * \mathrm{y}^{\prime}\right)$

$\left.\geq \lambda(\mathrm{x}) \cdot \lambda\left(\mathrm{x}^{\prime}\right)\right) \cdot\left(\mu(\mathrm{y}) \cdot \mu\left(\mathrm{y}^{\prime}\right)\right)$

$(\lambda(\mathrm{x}) \cdot \mu(\mathrm{y})) \cdot\left(\lambda\left(\mathrm{x}^{\prime}\right) \cdot \mu\left(\mathrm{y}^{\prime}\right)\right)$

$=(\lambda \times \mu)(x, y) \cdot(\lambda \times \mu)\left(x^{\prime}, y^{\prime}\right)$ 
Hence $\lambda \times \mu$ is a fuzzy $\operatorname{dot} B H$-ideal of $X \times X$.

Theorem 4.14. Let $\sigma$ be a fuzzy subset of a $B H$-algebra $X$ and $\mu_{\sigma}$ be the strongest fuzzy $\sigma$-relation on $B H$-algebra $\mathrm{X}$. Then $\sigma$ is a fuzzy dot $B H$-ideal of $X$ if and only if $\mu_{\sigma}$ is a Fuzzy dot $B H$-ideal of $X \times X$.

Proof. Assume that $\sigma$ is a fuzzy dot $\mathrm{BH}$-ideal of $\mathrm{X}$. For any $\mathrm{x}, \mathrm{y} \in \mathrm{X}$ we have $\mu_{\sigma}(0,0)=\sigma(0) \cdot \sigma(0) \geq \sigma(x) \cdot \sigma(y)=\mu_{\sigma}(x, y)$.

Let $x, x^{\prime}, y, y^{\prime} \in X$. Then

$$
\begin{gathered}
\mu_{\sigma}\left(\left(x, x^{\prime}\right) *\left(y, y^{\prime}\right)\right) \cdot \mu_{\sigma}\left(y, y^{\prime}\right) \\
=\mu_{\sigma}\left(x * y, x^{\prime} * y^{\prime}\right) \cdot \mu_{\sigma}\left(y, y^{\prime}\right) \\
=\left(\sigma(x * y) \cdot \sigma\left(x^{\prime} * y^{\prime}\right)\right) \cdot\left(\sigma(y) \cdot \sigma\left(y^{\prime}\right)\right) \\
=(\sigma(x * y) \cdot \sigma(y)) \cdot\left(\sigma\left(x^{\prime} * y^{\prime}\right) \cdot \sigma\left(y^{\prime}\right)\right) \\
\leq \sigma(x) \cdot \sigma\left(x^{\prime}\right)=\mu_{\sigma}\left(x, x^{\prime}\right) \cdot A n d, \\
\mu_{\sigma}\left(\mathrm{x}, x^{\prime}\right) \cdot \mu_{\sigma}\left(y, y^{\prime}\right)=\left(\sigma(x) \cdot \sigma\left(\mathrm{x}^{\prime}\right)\right) \cdot\left(\sigma(y) \cdot \sigma\left(\mathrm{y}^{\prime}\right)\right) \\
\left.=(\sigma(x) \cdot \sigma(y)) \cdot\left(\sigma(\mathrm{x})^{\prime}\right) \cdot \sigma\left(\mathrm{y}^{\prime}\right)\right) \\
\leq \sigma(x * y) \cdot \sigma\left(x^{\prime} * y^{\prime}\right) \\
=\mu_{\sigma}\left(x * y, x^{\prime} * y^{\prime}\right)=\mu_{\sigma}\left(\left(x, x^{\prime}\right) *\left(y, y^{\prime}\right)\right) .
\end{gathered}
$$

Thus $\mu_{\sigma}$ is a fuzzy dot $B H$-ideal of $X \times X$.

Conversely suppose that $\mu_{\sigma}$ is a fuzzy dot $B H$-ideal of $X \times X$. From (FBH1) we get

$(\sigma(0))^{2}=\sigma(0) \cdot \sigma(0)=c(0,0)$

And so $\sigma(0) \geq \sigma(x)$ for all $x \in X$. Also we have

$$
\begin{aligned}
(\sigma(\mathrm{x}))^{2} & =\mu_{\sigma}((\mathrm{x}, \mathrm{x}) \\
& \geq \mu_{\sigma}((\mathrm{x}, \mathrm{x}) *(\mathrm{y}, \mathrm{y})) \cdot \mu_{\sigma}(\mathrm{y}, \mathrm{y}) \\
& =\mu_{\sigma}((\mathrm{x} * \mathrm{y}),(\mathrm{x} * \mathrm{y})) \cdot \mu_{\sigma}(\mathrm{y}, \mathrm{y}) \\
=((\sigma(\mathrm{x} & * \mathrm{y}) \cdot \sigma(\mathrm{y})))^{2}
\end{aligned}
$$

Which implies that $\sigma(x) \geq \sigma(x * y) \cdot \sigma(y)$ for all $x, y \in X$.

Also we have

$$
\begin{aligned}
\left(\sigma(x * y)^{2}\right. & =\mu_{\sigma}((x * y), x * y) \\
& =\mu_{\sigma}((x, x) *(y, y)) \geq \mu_{\sigma}(x, x) \cdot \mu_{\sigma}(y, y) \\
& =(\sigma(x) \cdot \sigma(y))^{2}
\end{aligned}
$$

So $\sigma(x * y) \geq \sigma(x) \cdot \sigma(y)$ for all $x, y \in X$.

Therefore $\sigma$ is a fuzzy dot $B H$-ideal of $X$.

Proposition 4.15. Let $\mu$ be a left fuzzy relation on a fuzzy subset $\sigma$ of a $B H$-algebra $X$. If $\mu$ is a fuzzy dot BH-ideal of $X \times X$, then $\sigma$ is a fuzzy dot BH-ideal of a BH-algebra $\mathrm{X}$.

Proof.

Suppose that a left fuzzy relation $\mu$ on $\sigma$ is a fuzzy dot BH-ideal of $X \times X$.

Then

$\sigma(0)=\mu(0, z) \forall z \in X$

By putting $z=0$

$\sigma(0)=\mu(0,0) \geq \mu(x, y)=\sigma(x)$,

For all $x \in X$.

For any $x, x^{\prime}, y, y^{\prime} \in X$ 


$$
\begin{aligned}
& \sigma(x)=\mu(x, y) \geq \mu\left((x, y) *\left(x^{\prime}, y^{\prime}\right)\right) \cdot \mu\left(x^{\prime}, y^{\prime}\right) \\
& \left.=\mu\left(\left(x * x^{\prime}\right),\left(y * y^{\prime}\right)\right) \cdot \mu\left(y, y^{\prime}\right)\right) \\
& =\sigma\left(x * x^{\prime}\right) \cdot \sigma\left(x^{\prime}\right)
\end{aligned}
$$

Also

$$
\begin{aligned}
& \sigma(\mathrm{x} * \mathrm{x}) .=\mu\left(\mathrm{x} * \mathrm{x}^{\prime}, \mathrm{y} * \mathrm{y}^{\prime}\right)=\mu\left((\mathrm{x}, \mathrm{y}) *\left(\mathrm{x}^{\prime}, \mathrm{y}^{\prime}\right)\right) \\
& \geq \mu(\mathrm{x}, \mathrm{y}) \cdot \mu\left(\mathrm{x}^{\prime}, \mathrm{y}^{\prime}\right) \\
& =\sigma(\mathrm{x}) . \sigma\left(x^{\prime}\right)
\end{aligned}
$$

Thus $\sigma$ is a fuzzy dot BH-ideal of a BH-algebra X.

\section{REFERENCES}

[1] Y. Imai and K. Iséki, "On Axiom Systems of Propositional Calculi XIV," Proceedings of the Japan Academy, Vol. 42, No. 1, 1966, pp. 19-22. doi:10.3792/pja/1195522169

[2] Séki, Kiyoshi. An algebra related with a propositional calculus. Proc. Japan Acad. 42 (1966), no. 1, 26--29. doi:10.3792/pja/1195522171.

[3] Iseki K and Tanaka S: "An introduction to theory of BCK-algebras", Math. Japan. 23(1978), 126.

[4] P. Bhattacharya and N. P. Mukherjee, Fuzzy relations and fuzzy groups, Inform. Sci. 36(1985), 267-282.

[5] O. G. Xi, Fuzzy BCK-algebras, Math. Japan. 36(1991), 935-942.

[6] L. A. Zadeh, Fuzzy sets, Information Control, 8(1965) 338-353.

[7] K. Iseki and S. Tanaka, Ideal theory of BCK-algebras, Math. Japonica, 21(1976), 351-366.

[8] A. Rosenfeld, Fuzzy groups, J. Math. Anal. Appl. 35 (1971), 512-517.

[9] Y. B. Jun, E. H. Roh and H. S. Kim, On BH-algebras, Scientiae Mathematician 1(1) (1998), 347-354.

[10] Q. Zhang, E. H. Roh and Y. B. Jun, on fuzzy BH-algebras, J. Huanggang Normal Univ. 21(3) (2001), 14-19.

[11] J. Neggers and H. S. Kim, on $d$-algebras, Math. Slovaca, 49 (1996), 19-26.

[12] M. Akram, on fuzzy $d$-algebras, Punjab University Journal of Math.37 (2005), 61-76. 